\title{
Promoting immunization resiliency in the digital information age
}

\author{
Noni E MacDonald ${ }^{1 *}$, Eve Dubé2
}

\begin{abstract}
The avalanche of online information on immunization is having a major impact on the percentage of the population who choose to get vaccinated. Vaccine misinformation spreads widely with the interactive Web 2.0 and social media; this can bury science-based information. A plethora of immunization misinformation online is affecting trust in health care professionals and in public immunization programs. There are no simple solutions to this, but seven evidence-based strategies can help. First, listen to patients' and parents' concerns, and demonstrate responsiveness by adopting best immunization practices, such as pain mitigation. Second, recognize and alert others to anti-immunization tactics, namely, conspiracy theories, fake experts, selectivity, demands that vaccines be $100 \%$ safe and effective, misrepresentation and false logic. Third, avoid unproductive debates with those who have strongly held views, both in person and when using social media. Be respectful, stick to your key message, identify where to find useful information and exit. Fourth, consider establishing an attractive, easily searchable online presence that reflects the complex art of persuasion. Emphasize the benefits of vaccine, use reader-friendly graphics and highlight facts with stories to strengthen your case. Fifth, work with social media platform providers, not to stifle freedom of expression, but to help ensure that misinformation is not favoured in searches. Sixth, promote curriculum development in the schools to improve students' understanding of the benefits and safety of immunization and to foster critical thinking skills. To do this, optimize the use of age-appropriate comics and interactive learning tools such as electronic games. Seventh, to shift the narrative in specific communities with low vaccination rates, work with community leaders to build tailored programs that foster trust and reflect local values.
\end{abstract}

This work is licensed under a Creative Commons Attribution 4.0 International License.

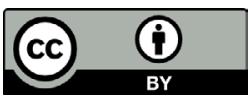

Affiliations

1 Department of Paediatrics Dalhousie University, IWK Health Centre Halifax, NS

2 Quebec National Institute of Public Health, Quebec City, QC

\section{*Correspondence: \\ noni.macdonald@dal.ca}

Suggested citation: MacDonald NE, Dubé E. Promoting immunization resiliency in the digital information age. Can Commun Dis Rep 2020;46(1):20-4. https://doi.org/10.14745/ccdr.v46i01a04

Keywords: immunization, vaccine, misinformation, social media, evidence-based strategies, education, vaccine hesitancy, Web 2.0, immunization resiliency, health communication

\section{Introduction}

The decision by individuals and parents to accept a vaccine is influenced by many factors. These can vary with time, place, vaccine and context (1). In Canada, as in other countries around the globe, the online immunization information avalanche is having a major impact on uptake. In the early digital age, public health information available online was static "read only" materials; with Web 2.0, online information has evolved into multidirectional, user-generated communication characterized by participation, collaboration and openness. Web 2.0 and social media have become the major modern platform for self-directed learning-a bottom-up approach with users reaching out, rather than a top-down strategy with experts providing information.

Most Canadians seek health information online, including information on immunization. This includes many seniors even though they came of age long before Web 2.0 (2). Unfortunately, only some of the immunization information on Web 2.0 and social media is science or evidence-based. Much online information is opinion or speculation as well as dramatic, often untrue but oft-repeated stories about adverse events presented as "alternative facts." Such vaccine misinformation can spread widely on social media (3), burying science-based information. Exchanges may spread widely, and comments may be vigorous and may become increasingly polarized overtime (4).

Exposure to immunization misinformation and fake news is now very common. A 2018 study from the United Kingdom found that over $40 \%$ of parents had been exposed to negative vaccine messages on social media (5). This is not a trivial issue: it poses a risk of confirmation bias (6). Confirmation bias occurs when people seek, select and retain the information that confirms their existing beliefs; it results in a bias in how new information is evaluated. Furthermore, with social media platforms, people are now being exposed to messages that are critical of vaccines even 
when they are not seeking this type of information. Negative vaccine information may elicit and/or reinforce vaccine hesitancy. It has been shown that as little as 5 to 10 minutes on an anti-vaccine website can negatively influence decisions to accept a vaccine (7).

A small percentage of people are profoundly anti-vaccine and refuse all vaccines (8). Some are very active online and have very firm opinions that evidence and facts are unlikely to shift. They may see themselves as experts, although this expertise is based upon misinformation gathered online from others holding similar world views. They are often only too willing to share their "knowledge" through YouTube videos, Twitter, Facebook groups and websites that readily pop up on Google searches. Such evergrowing misinformation can negatively influence vaccine decisions (9) and affect reporting of adverse events (10).

The digital information age has also changed the patient-health care professional relationship. Shared decision making is becoming normative and "top-down" paternalism is disappearing. This is not invariably negative; shared decision making is good when supported by sound scientific evidence that is shared and understood, but it is not good when decisions are influenced by misinformation. And the plethora of immunization misinformation online is not only influencing decision making; it is also affecting trust in health care professionals and in public immunization programs. Sadly, trolling for and subsequently online bullying of evidence-based vaccine experts by those with polarized opposite views, is an increasingly recognized problem $(11,12)$.

To address these challenges, "immunization resiliency" needs to be promoted. Immunization resiliency in this context means ensuring that vaccination programs are adapted to the current digital communication environment in order to grow public trust in health care providers and optimize vaccine acceptance over time. The objective of this article is to identify best practices for front line vaccine providers in order to promote vaccine resiliency. This is the first of a series of articles, produced by The Canadian Vaccination Evidence Resource and Exchange Centre (CANVax), which include both the identification of existing resources and the creation of new resources by a group of multidisciplinary professionals (13).

\section{What can health care professionals do to promote immunization resiliency?}

There are no simple solutions to addressing the avalanche of digital misinformation about vaccines and the deleterious effects this has caused, but evidence-based strategies can help. We highlight seven key strategies that are based on reviews of psychological research on persuasion, myth debunking, science denialism, communication science and research on impact of social media.

\section{Listen to patients and parents}

In this postpaternalism world, it is important to learn what the issues of concern are when interacting with people who are contemplating vaccination both at an individual and at a community level. At the clinical level, health care providers need to be prepared to listen and then address concerns as they arise. Using the mini-motivational interviewing tools can be effective in moving the patient towards vaccine acceptance (14). If pain on immunization is a concern-and it is for over $40 \%$ of mothers of infants needing immunization-then use best practices for mitigation pain based upon Canada's 2015 guidelines (15).

Frontline health care providers need to be encouraged to report to their local public health unit common concerns they are hearing. Immunization programs can use these concerns as well as those found through analysis of social media to develop targeted communication messages using traditional and social media (16). There is a good rationale for directly countering social media misinformation because of its potential influence. Effective strategies to do this have been proposed (17). Remember: data tells but stories sell-be succinct and straightforward.

\section{Recognize and alert others to anti-immunization tactics}

Recognize the tactics often used by those opposing immunization: conspiracy theories, fake experts, selectivity, demands that vaccines be $100 \%$ safe and effective,

misrepresentation and false logic (18). Correcting misinformation and highlighting these techniques being used can help inoculate against misinformation (19). Draw attention to these, especially in a forum where there is a bigger audience, for example, in a Facebook group, etc., and only if your message is not so polarized that it will be buried. Alerting to these tactics and correcting misinformation can be salutary for those without confirmed negative views on immunization (20). Remember your target is not the person promulgating the misinformation but the reader of the misinformation.

\section{Avoid unproductive debates and be respectful}

Be aware that many online anti-vaccine forums are very polarized; joining in may not be helpful (4). While the evidence is clear that vaccines are safe and effective and that diseases with serious consequences-even death-can occur when immunization is omitted, this will not convince those with strongly held opposing views. Do not fall into the persuasion loop trap. When your efforts to correct repeated examples of selectivity or misrepresentation are met with "yes, but," this can lead to a never-ending dialogue. It is more effective to be respectful, stick to your message, identify where to find more information and exit the conversation, that is, limit direct engagement with those who have strong anti-vaccination views, either online or in person. Repeating their arguments as you attempt to refute them can be counterproductive as the misinformation may stick (21). 
When you do engage in social media opportunities, do so wisely. Tailored and targeted messages and information can help shape attitudes and improve uptake $(22,23)$. As noted above, it can be useful to alert others to anti-vaccination tactics in public forums $(20,21)$. Get your point across succinctly_remember that stories are often more powerful than dry facts and numbers-and steer readers to reputable sites for more quality information.

Never be disparaging or demeaning in public or to a patient. The term "vaccine deniers" has been used as convenient shorthand, but a more neutral description, such as "those with strong antivaccine opinions," may be better received during discussions.

\section{Consider developing an attractive, easily searchable online presence}

When health professionals and/or their organizations have a presence on the internet, presenting evidence-based information about immunization online can be helpful. However, avoid "knowledge dumps" or posting large quantities of very technical information to try and counter every new piece of misinformation. Be proactive: promote positive messages about vaccination in a succinct and easy-to-grasp manner.

If you plan to build a pro immunization website, make it appealing, easily searchable and as interactive as possible. Clearly identify your evidence-based key messages, with short reader-friendly text. The complex art of persuasion calls for a blend of different strategies, including the need for a straightforward presentation of the "what", "how", "where", "when" and "who" complemented with stories. Colour and graphics also help to increase the impact of key points (24).

Static websites are dated. If resources permit, include a place where visitors can post questions. Having nowhere to make inquiries can be frustrating. If you do not have the resources for this, consider including a common question-and-answer information sheet or provide a link to a reliable resource with a query service, such as the Canadian Immunization Guide (25). If you are able to respond to queries, avoid entering into protracted debates with anyone who has strong anti-vaccination views.

\section{Alert social media platform providers to misinformation online}

Work with social media platform providers, not to stifle freedom of expression, but to help ensure that searches do not favour misinformation (26). As an example, Facebook has started removing extreme misinformation websites (27). Consider learning from the techniques being used to help control online hate websites and forums (28).

\section{Promote immunization and science literacy curriculum development for use in schools}

Work with departments of education to help develop curricula that can improve students' understanding of the benefits and safety of immunization and risks of vaccine-preventable disease. Online games, comics, animation and other forms of visual communication may have more appeal than static pages. While many groups in Canada and elsewhere are developing online immunization educational materials for students (29), it is important to ensure that these fit the context of the audience. For example, the British Columbia Centre for Disease Control education website Kids Boost Immunity (30), which is formatted as an interactive quiz, is evidence based, educational and well-liked by students. Evaluations and assessments have shown that children learn about immunization from the site.

A vaccine-specific curriculum is not enough to help increase immunization resiliency. Students also need well-developed critical thinking skills and much higher levels of science and digital literacy if they are to see through the misinformation and con-artist techniques in use on the internet. These skills are needed to assess more than just immunization information, but immunization makes an excellent case. There are resources available to help with this, for example, Media Smarts (31).

\section{Work with community leaders to build tailored programs in specific communities}

Vaccine uptake may be lower in some communities than in others (32). Listen to and work with communities and with community leaders to build trust in immunization through multipronged campaigns (which may involve online media) that explicitly support local values and are respectful of their context $(33,34)$. Tailored programs can help shift the narrative in the community.

Beyond targeting subgroups, pulling together a collaborative network of national/provincial immunization programs, academia, health professional societies, health centres and health authorities, who add their voices to the positive message of the importance of vaccines and safety, can help sway public attitudes. This is known as the "gateway belief" (35). These common messages also save time and support frontline health professionals' positive recommendations for immunization.

\section{Conclusion}

Misinformation and polarization of online immunization information is not going to go away. For clinical care and public health, the work to increase immunization resiliency is only going to become even more complex. Health care and public health professionals need to better understand why misinformation is so appealing and why polarization is to be expected. People do need to be listened to. Immunization concerns need to be assuaged. Over time, we must become better at discerning what information is credible, be it about health, climate change, the economy or politics. While teaching critical thinking and digital and science literacy is a major step forward, we also need to focus on how better to connect with communities and how to 
reframe the immunization messages so they are more effectively heard and better appreciated. Useful accessible materials can be found are the National Advisory Committee on Immunization summary statements (for example, 36), the Canadian Immunization Guide (25), the immunization section on the Caring For Kids Website of the Canadian Paediatric Society (37), Immunize Canada (38) and the CANVax website (13).

\section{Authors' statement}

NM - Writing original draft

ED - Writing, review and editing

\section{Conflict of interest}

Dr. MacDonald reports grants from the Public Health Agency of Canada, the Canadian Institutes of Health Research, Nova Scotia Health Authority, IWK Health Authority and the Canadian Immunization Research Network, Dr Dubé reports grants from the Public Health Agency of Canada, the Quebec Ministry of Health and Social Services, le Fonds de la recherche en Santé du Québec, the Canadian Institutes of Health Research, the Canadian Immunization Research Network, and the Social Sciences and Humanities Research Council of Canada.

\section{Acknowledgements}

Contributions to CANVax come from a very wide range of authors, committees, immunization partners, reviewers and especially the CANVax secretariat at Canadian Public Health Association.

\section{References}

1. MacDonald NE; SAGE Working Group on Vaccine Hesitancy. Vaccine hesitancy: Definition, scope and determinants. Vaccine 2015 Aug;33(34):4161-4. DOI PubMed

2. Paula A. A day in the life: How do older Canadians spend their time? March 21, 2018. Sta-tistics Canada. (Accessed 2019-11-2). https://www150.statcan.gc.ca/n1/pub/75006-x/2018001/article/54947-eng.htm

3. Bauch CT, Galvani AP. Epidemiology. Social factors in epidemiology. Science 2013 Oct;342(6154):47-9. DOI PubMed

4. Meyer SB, Violette R, Aggarwal R, Simeoni M, MacDougall $\mathrm{H}$, Waite N. Vaccine hesitancy and Web 2.0: exploring how attitudes and beliefs about influenza vaccination are exchanged in online threaded user comments. Vaccine 2019 Mar;37(13):1769-74. DOl PubMed

5. Royal Society for Public Health. Moving the needle: promoting vaccination uptake across the life course. London (UK): Royal Society for Public Health; 2018 Dec. https://www. rsph.org.uk/uploads/assets/uploaded/f8cf580a-57b5-41f48e21de333af20f32.pdf
6. Meppelink CS, Smit EG, Fransen ML, Diviani N. "I was right about vaccination": confirmation bias and health literacy in online health information seeking. J Health Commun 2019;24(2):129-40. DOI PubMed

7. Betsch C, Renkewitz F, Betsch T, Ulshöfer C. The influence of vaccine-critical websites on perceiving vaccination risks. J Health Psychol 2010 Apr;15(3):446-55. DOI PubMed

8. Dubé E, Bettinger JA, Fisher WA, Naus M, Mahmud SM, Hilderman T. Vaccine acceptance, hesitancy and refusal in Canada: challenges and potential approaches. Can Commun Dis Rep 2016;42(12):246-51. DOl PubMed

9. Dunn AG, Surian D, Leask J, Dey A, Mandl KD, Coiera E. Mapping information exposure on social media to explain differences in HPV vaccine coverage in the United States. Vaccine 2017 May;35(23):3033-40. DOl PubMed

10. Faasse K, Porsius JT, Faasse J, Martin LR. Bad news: the influence of news coverage and Google searches on Gardasil adverse event reporting. Vaccine 2017 Dec;35 49 Pt B:68728. DOI PubMed

11. Broniatowski DA, Jamison AM, Qi S, AlKulaib L, Chen T, Benton A, Quinn SC, Dredze M. Weaponized Health communication: twitter bots and Russian trolls amplify the vaccine debate. Am J Public Health 2018 Oct;108(10):137884. DOI PubMed

12. Starbird K. Disinformation's spread: bots, trolls and all of us. Nature 2019 Jul;571(7766):449. DOl PubMed

13. CANVax. The Canadian Vaccination Evidence Resource and Exchange Centre. Ottawa (ON). https://www.canvax.ca/

14. Gagneur A, Gosselin V, Dubé È. Motivational interviewing: A promising tool to address vaccine hesitancy. Vaccine 2018 Oct;36(44):6553-5. DOI PubMed

15. Taddio A, McMurtry CM, Shah V, Riddell RP, Chambers CT, Noel M, MacDonald NE, Rogers J, Bucci LM, Mousmanis P, Lang E, Halperin SA, Bowles S, Halpert C, Ipp M, Asmundson GJ, Rieder MJ, Robson K, Uleryk E, Antony MM, Dubey V, Hanrahan A, Lockett D, Scott J, Bleeker EV; HELPinKids\&Adults. Reducing pain during vaccine injections: clinical practice guideline. CMAJ 2015 Sep;187(13):975-82. DOI PubMed

16. Müller MM, Salathé M. Crowd breaks: tracking health trends using public social media data and crowdsourcing. Front Public Health 2019 Apr;(7):81. DOI PubMed

17. Steffens MS, Dunn AG, Wiley KE, Leask J. How organisations promoting vaccination respond to misinformation on social media: a qualitative investigation. BMC Public Health 2019 Oct;19(1):1348. DOI PubMed

18. MacDonald NE. When science meets Google: reflections on research and evidence in the age of science deniers. Clin Invest Med 2018 Nov;41 suppl:35-7. DOI PubMed

19. van der Linden S, Maibach E, Cook J, Leiserowitz A, Lewandowsky S. Inoculating against misinformation. Science 2017 Dec;358(6367):1141-2. PubMed 
20. Schmid $P$, Betsch $C$. Effective strategies for rebutting science denialism in public discussions. Nat Hum Behav 2019 Sep;3(9):931-9. DOI PubMed

21. Cook J, Lewandowsky S, Ecker UK. Neutralizing misinformation through inoculation: exposing misleading argumentation techniques reduces their influence. PLoS One 2017 May;12(5):e0175799. DOI PubMed

22. Glanz JM, Wagner NM, Narwaney KJ, Kraus CR, Shoup JA, Xu S, O'Leary ST, Omer SB, Gleason KS, Daley MF. Web-based social media intervention to increase vaccine acceptance: a randomized controlled trial. Pediatrics 2017 Dec;140(6):e20171117. DOl PubMed

23. Mohanty S, Leader AE, Gibeau E, Johnson C. Using Facebook to reach adolescents for human papillomavirus (HPV) vaccination. Vaccine 2018 Sep;36(40):5955-61. DOI PubMed

24. Garett R, Chiu J, Zhang L, Young SD. A literature review: website design and user engagement. Online J Commun Media Technol 2016 Jul;6(3):1-14. DOI PubMed

25. Public Health Agency of Canada. Canadian immunization guide. Ottawa (ON): Govern-ment of Canada (Accessed 2019-11-03). https://www.canada.ca/en/public-health/ services/canadian-immunization-guide.html

26. Allcott $\mathrm{H}$, Gentzkow M, Yu C. Trends in the diffusion of misinformation on social media. Cambridge (MA): National Bureau of Economic Research, Inc.; 2019 (Accessed 201905-09). NBER Working Papers 25500. https://web.stanford. edu/ gentzkow/research/fake-news-trends.pdf

27. Yurieff K, Kelly H. Facebook makes changes in its ongoing attempt to limit misinformation. New York (NY): CNN Business (Accessed 2019-11-03). https://www.cnn. com/2019/04/10/tech/facebook-integrity-updates/index.html

28. Johnson NF, Leahy R, Restrepo NJ, Velasquez N, Zheng M, Manrique $P$, Devkota $P$, Wuchty $S$. Hidden resilience and adaptive dynamics of the global online hate ecology. Nature 2019 Sep;573(7773):261-5. DOI PubMed

29. Wilson K, Atkinson K, Crowcroft N. Teaching children about immunization in a digital age. Hum Vaccin Immunother 2017 May;13(5):1155-7. DOl PubMed
30. Immunity KB. Free Science, Social Studies and Health lessons developed by teachers to inspire digital-age students in support of UNICEF Canada! Victoria (BC): Public Health Association of BC (Accessed 2019-11-03). https:// kidsboostimmunity.com/

31. Smarts M. Break the Fake.ca. Ottawa (ON): Media Smarts; www.mediasmarts.ca

32. Wilson SE, Seo CY, Lim GH, Fediurek J, Crowcroft NS, Deeks SL. Trends in medical and nonmedical immunization exemptions to measles-containing vaccine in Ontario: an annual cross-sectional assessment of students from school years 2002/03 to 2012/13. CMAJ Open 2015 Jul;3(3):E317-23. DOl PubMed

33. Attwell K, Freeman M. I Immunise: an evaluation of a values-based campaign to change attitudes and beliefs. Vaccine 2015 Nov;33(46):6235-40. DOI PubMed

34. Dubé E, Leask J, Wolff B, Hickler B, Balaban V, Hosein E, Habersaat K. The WHO Tailor-ing Immunization Programmes (TIP) approach: review of implementation to date. Vaccine 2018 Mar;36(11):1509-15. DOI PubMed

35. van der Linden SL, Clarke CE, Maibach EW. Erratum to: Highlighting consensus among medical scientists increases public support for vaccines: evidence from a randomized ex-periment. BMC Public Health 2015 Dec;15:1207. DOI

36. Zhao L, Young K, Gemmill I on behalf of the National Advisory Committee on Immuniza-tion (NACl). Summary of the NACI Seasonal Influenza Vaccine Statement for 2019-2020. Can Commun Dis Rep 2019;45(6):149-55. DOI PubMed

37. Caring for kids: information for parents from Canada's paediatricians. Immunization. Ot-tawa (ON): Canadian Paediatric Society. https://www.caringforkids.cps.ca/ handouts/immunizations-index

38. Immunize Canada. Immunization protects individuals and communities by preventing the spread of disease. Ottawa (ON): Canadian Public Health Association. https://www. immunize.ca/ 